\title{
FATORES ASSOCIADOS AO BAIXO PESO AO NASCER
}

\author{
Emília Coutinho \\ Escola Superior de Saúde de Viseu (ecoutinhoessv@gmail.com); \\ Lúcia Araújo \\ Centro Hospitalar Tondela-Viseu \\ Carlos Pereira* \\ João Duarte* \\ Paula Nelas* \\ Cláudia Chaves* \\ *Escola Superior de Saúde de Viseu \\ https://doi.org/10.17060/ijodaep.2016.n2.v1.229
}

Fecha de Recepción: 24 Julio 2016

Fecha de Admisión: 1 Octubre 2016

\section{RESUMEN}

Enquadramento: 0 baixo peso ao nascer é um fator de risco para a morbi-mortalidade neonatal, e um indicador geral do nível de saúde de uma população. Associa-se a baixos níveis de desenvolvimento socioeconómico, características maternas e de assistência materno-infantil.

Objetivos: Analisar a influência das variáveis sociodemográficas, obstétricas e de assistência pré-natal ao baixo peso ao nascer.

Participantes: 1846 puérperas, em que 161 apresentam recém-nascidos de baixo peso ao nascer.

Métodos: Estudo transversal, descritivo correlacional e prospetivo com uma amostra não probabilística intencional. Foi utilizado um questionário aplicado entre Março de 2010 e Maio de 2012 , em 26 instituições de saúde públicas portuguesas.

Resultados: 0 baixo peso ao nascer, foi associado a: idade gestacional $\geq 41$ semanas $(p<0,001)$, idade materna de risco*número gestações de risco $(p=0,021)$; baixa escolaridade* rendimentos $\leq 1000 €(p=0,036)$, baixa escolaridade ${ }^{*}$ desemprego $(p=0,044)$, número filhos de risco*idade gestacional de termo $(p<0,001)$, patologias na gravidez $z^{*}$ idade gestacional de termo $(p<0,001)$, patologias prévias ${ }^{*}$ idade gestacional de termo $(p<0,001)$, e com erro de $10 \%$ à baixa escolaridade $(p=0,074)$ e não planeamento da gravidez ( $p=0,089$ ). A prevalência de BPN foi de $8,7 \%$. 


\section{FATORES ASSOCIADOS AO BAIXO PESO AO NASCER}

Conclusão: A determinação dos fatores que favorecem 0 aparecimento do baixo peso ao nascer é fundamental para o planeamento e desenvolvimento de ações no âmbito da saúde maternoinfantil.

Palavras-chave: Baixo Peso ao Nascer; Recém-nascido; Fatores de Risco; Fatores Sociodemográficos; Assistência pré-natal; Gravidez.

\section{ABSTRACT}

\section{Factors associated with low birthweight}

Framework: The low birthweight is a risk factor for neonatal morbidity and mortality and a general indicator of health status of a population. It can be associated to low levels of socioeconomic development, prenatal care and maternal charateristics.

Objectives: Analyze the influence of sociodemographic, obstetric and prenatal care variables to low birthweight.

Participants: 1846 post-partum women, where only 161 had newborns with low birthweight.

Methods: This is a wide-ranging, descriptive-correlacional and forward-looking study with a non-probable intentional sample. It was used a questionnaire, applied between March 2010 and May 2012, in 26 Portuguese institutions of public health.

Results: The low birthweight was associated: $\geq 41$ weeks $(p=0,000)$, without obstetric risk $(p=0,001)$, without obstetric risk/unplanned pregnancy $(p=0,036)$, obstetric risk/maternal age risk $(p=0,007)$, without sociodemographic risk/gestacional age without risk $(p=0,000)$, sociodemographic risk/gestacional age without risk $(p=0,007)$, risk on prenatal care/maternal age risk $(p=0,020)$, prenatal care without risk/gestacional age without risk $(p=0,000)$, maternal age risk/risk number of pregnancies $(p=0,021)$, low education/income $\leq 1000 €(p=0,036)$, low education/unemployment $(p=0,044)$, risk number of children/gestational age without risk $(p=0,000)$, pregnancy diseases /gestational age without risk $(p=0,000)$, previous diseases/gestational age without risk $(p=0,000)$, and with a margin error of $10 \%$, low education $(p=0,074)$ and unplanned pregnancy $(p=0,089)$. The prevalence of LBW was $8.7 \%$.

Conclusion: The determination of the factors that contribute to the appearance of low birthweight is essential for the planning, implementation and development of actions related to maternal and children health.

Key-words: Low Birth Weight, Newborn, Risk Factors, Sociodemographic Factors; Prenatal care, Pregnancy.

\section{INTRODUÇÃO}

A gravidez é uma fase extremamente importante para a vida da maior parte das mulheres, contudo não deixa de ser um período em que são múltiplos os fatores que podem interferir de forma direta ou indireta no seu desfecho e por consequência influenciar no peso ao nascer daquela criança. Dentro desses fatores destacam-se os maternos, socioeconómicos, genéticos, placentários, nutricionais, hormonais ou ambientais (Kramer, 1987; E. S. N. Silva \& Lahorgue, 2005).

Segundo a definição da Organização Mundial da Saúde (OMS), o baixo peso ao nascer (BPN) corresponde a um peso $\leq 2499 \mathrm{~g}$ do recém-nascido aquando do nascimento, independentemente da sua idade gestacional (Eisenhauer, Uddin, Albers, Paton, \& Stoughton, 2011).

A temática do BPN surge como de extrema importância, uma vez que entre ela e a mortalidade e morbilidade infantil existem evidências de interligação. Efetivamente alguns estudos indicam que a mortalidade neonatal é 20 vezes maior nos recém-nascidos de BPN e 200 vezes maior nos que apresentam um muito baixo peso $(\leq 1499 \mathrm{~g})$ quando comparados aos de peso normal (Ohlsson \& Shah, 2008). 
O BPN é uma questão de saúde pública com um impacto significativo individual, familiar e social (Shah, 2010). Os recém-nascidos com esse peso apresentam alterações do vínculo, diminuição do período de tempo de amamentação, maior risco de desenvolvimento de patologias crónicas durante a sua vida adulta, uma maior propensão a atrasos de desenvolvimento e dificuldades de aprendizagem, o que acarretará tanto para a família, como para a sociedade, onde estão inseridos um custo elevado (Ohlsson \& Shah, 2008).

A redução da prevalência do BPN não tem sido fácil, e apesar da extensiva pesquisa sobre a sua etiologia, esta ainda permanece desconhecida em cerca de 30\% dos casos clínicos (Vettore, Gama, Lamarca, Schilithz, \& Leal, 2010).

Mundialmente, entre os anos 2000-2009, a taxa média de BPN foi de $14 \%$, sendo que Portugal apresentou um percentual de $8 \%$. Contudo é nos países em desenvolvimento que essa taxa apresenta valores mais elevados ( $24 \%$ na região sudeste), onde a restrição do crescimento intrauterino (RCIU) se torna o principal causador desse acontecimento, ao invés do nascimento pré-termo que é o principal responsável nos países desenvolvidos (WHO, 2011).

Por conseguinte, o baixo peso ao nascer pode ser atribuído aos dois fenómenos, ou combinação entre ambos nomeadamente RCIU e parto pré-termo (Carniel, Zanolli, Antônio, \& Morcillo, 2008). Quando estes dois fatores se associam, tornam o BPN no mais importante indicador de mortalidade neonatal, perinatal e infantil (Kilsztajn, Rossbach, Carmo, \& Sugahara, 2003).

Vários estudos têm sido realizados na tentativa de identificar os fatores desencadeantes do aparecimento do baixo peso ao nascer. Das características mais prevalentes surgem as demográficas, socioeconómicas, maternas, obstétricas, de assistência pré-natal e comportamentais (Lasker, Coyle, Li, \& Ortynsky, 2005). Outros estudos têm referido a relação do BPN com as características paternas, ambientais, em casos de violência, situações de stress, e depressão durante a gravidez (Ohlsson \& Shah, 2008).

Pela importância da deteção dos fatores associados ao BPN para o indivíduo, família e sociedade e pela relevância do papel preponderante que 0 enfermeiro especialista em saúde materna e obstetrícia poderá ter na sua prevenção, justifica-se a realização deste estudo 0 qual pretende estudar a influência de variáveis sociodemográficas, obstétricas e de assistência pré-natal no BPN e prevalência de crianças com BPN.

\section{MATERIAL E MÉTODOS}

0 tipo de investigação delineada foi uma investigação não experimental, de análise quantitativa, transversal, descritivo correlacional e prospetiva.

A amostra desta investigação é do tipo não probabilística intencional e resultou da aplicação de um questionário a 1846 puérperas portuguesas, internadas em 26 maternidades de Portugal, entre os meses de Março de 2010 a Maio de 2012.

0 instrumento de recolha de dados aplicado encontra-se organizado em três partes: a primeira respeita a caracterização sociodemográfica, a segundo a vigilância da gravidez e a terceira a descrição obstétrica.

Do total da amostra destacam-se as seguintes características: 0 grupo etário entre os 20 e 34 anos é o mais prevalente (76,5\%), sendo que a média está centrada nos 29,95 anos. Um percentual de 66,8\% apresenta uma escolaridade até ao Ensino Secundário, 74,3\% encontram-se empregadas, e $61,8 \%$ auferem de um rendimento mensal superior a 1000 euros.

Com o intuito de minimizar divergências interpretativas e no sentido de se criar um índice de risco, procedemos à definição de alguns conceitos operacionais relacionados com as variáveis sociodemográficas, obstétricas e de assistência pré-natal.

A estratégia utilizada na construção das variáveis de risco teve em conta a revisão bibliográfica 


\section{FATORES ASSOCIADOS AO BAIXO PESO AO NASCER}

e a descrição das características das variáveis em classes, associando-as posteriormente em grupos de Risco ou Sem Risco (Tabela 1).

Tabela 1: Caracterização das variáveis segundo o risco

\begin{tabular}{|c|c|c|c|}
\hline Variáveis & & Risco & Sem Risco \\
\hline \multirow{4}{*}{$\begin{array}{l}\text { Variáveis } \\
\text { sociodemográficas }\end{array}$} & Idade & $\leq 19$ Anos e $\geq 35$ anos & 20-34 Anos \\
\hline & Escolaridade & Até ao Ens. Secundário & Ensino Superior \\
\hline & Situação Profissional & Desempregada & Empregada \\
\hline & Rendimento Mensal & $\leq 1000 €$ & $>1000 €$ \\
\hline \multirow{5}{*}{$\begin{array}{l}\text { Variáveis Assistência } \\
\text { Pré-natal }\end{array}$} & Planeamento da Gravidez & Não planeada & Planeada \\
\hline & Desejo da gravidez & Não desejada & Desejada \\
\hline & Vigilância da Gravidez & Não vigiada & Vigiada \\
\hline & Patologias Prévias & Sim & Não \\
\hline & Ganho Ponderal & $\leq 6 \mathrm{~kg}$ & $>6 \mathrm{~kg}$ \\
\hline \multirow{6}{*}{ Variáveis Obstétricas } & N. Gestações & Primípara; 4 ou + gestações & 2 a 3 gestações \\
\hline & N. Filhos & 1 filho e 4 ou + filhos & 2 a 3 filhos \\
\hline & Gemelaridade & Sim & Não \\
\hline & Intervalo Interparto & $\leq 2$ anos e $\geq 6$ anos & 3 a 5 anos \\
\hline & Peso 1 응 Filho & $\leq 2499 \mathrm{~g}$ & $>2500 \mathrm{~g}$ \\
\hline & Idade Gestacional & $<37$ e $\geq 41$ & 37- 40 semanas \\
\hline
\end{tabular}

\section{RESULTADOS}

Da descrição da totalidade da amostra, tendo em conta o peso do recém-nascido, verifica-se que $88,1 \%$ (1626 casos) apresentam um peso adequado, 8,7\% (161 casos) apresentam BPN e 3,2\% (59 casos) apresentam macrossomia.

Atendendo à variável BPN, os resultados estatísticos a salientar são: um mínimo de $650 \mathrm{~g}$ e um máximo de $2495 \mathrm{~g}$, a média centra-se nos $2117,86 \mathrm{~g}$, com um desvio padrão a oscilar em torno da média de $385,310 \mathrm{~g}$, com coeficiente de variação de $18,19 \%$.

Já de acordo com as variáveis sociodemográficas, a maior prevalência de BPN encontra-se nas puérperas do grupo etário materno $\leq 19$ anos $(p=0,001)$; escolaridade até ao secundário $(p=0,007)$; desempregada; rendimento mensal $\leq 1000 €(p<0,001)$ e a coabitarem sem companheiro $(p=0,007)$, onde também apresentam diferenças estatísticas significativas.

Segundo as variáveis de Assistência Pré-natal, as puérperas com maior incidência de BPN e diferença estatística bastante e altamente significativa são as puérperas que não desejaram $(p=0,004)$ nem vigiaram a gravidez adequadamente $(p=0,005)$, que apresentaram patologias durante a gravidez $(p<0,001)$ e mantiveram um hábito tabágico durante a mesma $(p<0,001)$. Com diferença estatística significativa surgem as puérperas com um ganho ponderal $\leq 6 \mathrm{~kg}(p=0,015)$.

De acordo com as variáveis obstétricas, a incidência maior de BPN surge nas que são primíparas, nas puérperas com 4 ou mais filhos, com gravidez gemelar, com intervalo interparto inferior ou igual a 2 anos ou ausência de intervalo por primiparidade, com filho anterior de BPN (primeiro filho), e parto em idade gestacional de pré-termo. 
Com diferenças estatísticas altamente significativas encontram-se: a gemelaridade $(p<0,001), 0$ peso do 10 filho $\leq 2499 \mathrm{~g}(p<0,001)$ e a idade gestacional inferior às 37 semanas $(p<0,001)$.

De acordo com a revisão bibliográfica, sobressai a interação de algumas variáveis, determinando um baixo peso à nascença. Nesse sentido foram efetuadas algumas interações com as variáveis: idade materna, escolaridade, rendimento mensal, vigilância da gravidez, patologias prévias e durante a gravidez, hábitos alcoólicos e tabágicos e número de filhos; com o intuito de determinar essa possibilidade de relação.

Da interação com a idade materna resultaram diferenças estatísticas, maioritariamente localizadas na Idade de Risco associada a: escolaridade de risco ( $p=0,004)$; situação profissional de risco $(p<0,001)$; rendimento mensal de risco $(p<0,001)$; número de gestações de risco $(p=0,005)$; número de filhos de risco $(p=0,033)$; planeamento de risco $(p=0,019)$; sem patologias prévias $(p=0,039)$; ganho ponderal de risco $(p=0,001)$; estado civil de risco $(p<0,001)$ e sem consumo de álcool $(p=0,057$ e residual $=2,5)$.

Nas que apresentam diferenças significativas com a Idade Sem Risco surgem: a vigilância de risco $(p=0,003)$; a gemelaridade $(p<0,001)$; com patologias da gravidez $(p<0,001)$ e 0 tabagismo $(p<0,001)$.

Na variável Escolaridade de Risco destacam-se a presença de diferenças estatísticas 0: rendimento mensal de risco ( $p=0,001)$; situação profissional de risco $(p<0,001)$; estado civil de risco $(p=0,003)$; vigilância da gravidez de risco $(p=0,003)$; número de filhos de risco $(p=0,033)$; intervalo interparto de risco $(p=0,020)$, tabagismo $(p<0,001)$ e ganho ponderal de risco $(p=0,004)$.

De acordo com o Rendimento Mensal de Risco, os cruzamentos efetuados apresentam diferenças estatísticas, no Ganho Ponderal de Risco ( $p=0,001)$; Estado Civil de risco e sem risco $(p<0,001)$ e Vigilância de risco $(p<0,001)$.

A Vigilância da gravidez de risco demonstrou diferenças estatísticas no: estado civil de risco $(p=0,003)$; planeamento de risco $(p=0,028)$; presença de patologias prévias $(p=0,002)$; presença de patologias na gravidez $(p<0,001)$; número de filhos de risco $(p=0,009)$; sem consumo de álcool $(p=0,027)$; consumo de tabaco $(p<0,001)$ e ganho ponderal inadequado $(p<0,001)$.

$\mathrm{Na}$ análise da interação das patologias durante a gravidez e prévias à gravidez obtiveram-se diferenças estatísticas altamente significativas na presença e ausência patologia da gravidez com idade gestacional de risco $(p=0,000)$ e na ausência ou presença de patologias na gravidez/ idade gestacional de risco $(p<0,001)$.

$\mathrm{Na}$ análise das interações com os hábitos tabágicos e alcoólicos surgem com diferenças estatísticas altamente significativas as relações Hábito Alcoólico ou sem hábito alcoólico/Patologias na gravidez $(p<0,001)$ e Hábito Tabágico/Ganho Ponderal de risco ou sem risco $(p<0,001)$.

$\mathrm{Na}$ interação da variável Número de filhos obteve-se como altamente significativa a relação: Número de Filhos de risco/Patologias na gravidez $(p<0,001)$ e N.․ de Filhos de risco ou sem risco/ Idade Gestacional de risco ( $p=0,000)$; e bastante significativa na N.․ Filhos de risco/Intervalo interparto de risco $(p=0,030)$.

A verificação da validade dos resultados obtidos da análise descritiva foi efetuada sob a aplicação de testes de U de Mann-Whitney e Kruskal-Wallis.

Da análise inferencial das variáveis e interações entre variáveis, somente demonstram apresentar relação com o BPN, as puérperas com baixa escolaridade, gravidez não planeada e parto na idade gestacional $\geq 41$ semanas; ainda as puérperas com idade materna de risco associada a número de gestações de risco; baixa escolaridade com rendimentos $\leq 1000 €$ e desemprego; número de filhos de risco e gestação de termo e presença de patologias prévias e na gravidez com idade gestacional de termo cf. tabela 2 e 3. 


\section{FATORES ASSOCIADOS AO BAIXO PESO AO NASCER}

Tabela 2: Teste U Mann-Whitney e Kruskal-Wallis entre variáveis independentes e o BPN

\begin{tabular}{l|l|l|l}
\hline Baixo peso ao nascer & OM & U & P \\
\hline Escolaridade & & & \\
$\leq 12$ - Ano & 84,78 & 1963,0 & 0,074 \\
Ensino Superior & 69,58 & & \\
Planeamento da Gravidez & 93,30 & 1706,0 & 0,089 \\
Não & 77,83 & $\mathrm{KW}$ & $\mathrm{P}$ \\
Sim & OM & & \\
\hline Baixo peso ao nascer & & 38,468 & 0,000 \\
\hline Idade Gestacional & 60,85 & & \\
$\leq 36$ sem & 106,29 & & \\
$37-40$ sem & 133,50 & & \\
$\geq 41$ sem & & \\
\hline
\end{tabular}

Tabela 3: Teste de Kruskal-Wallis entre a interação de variáveis independentes e o BPN

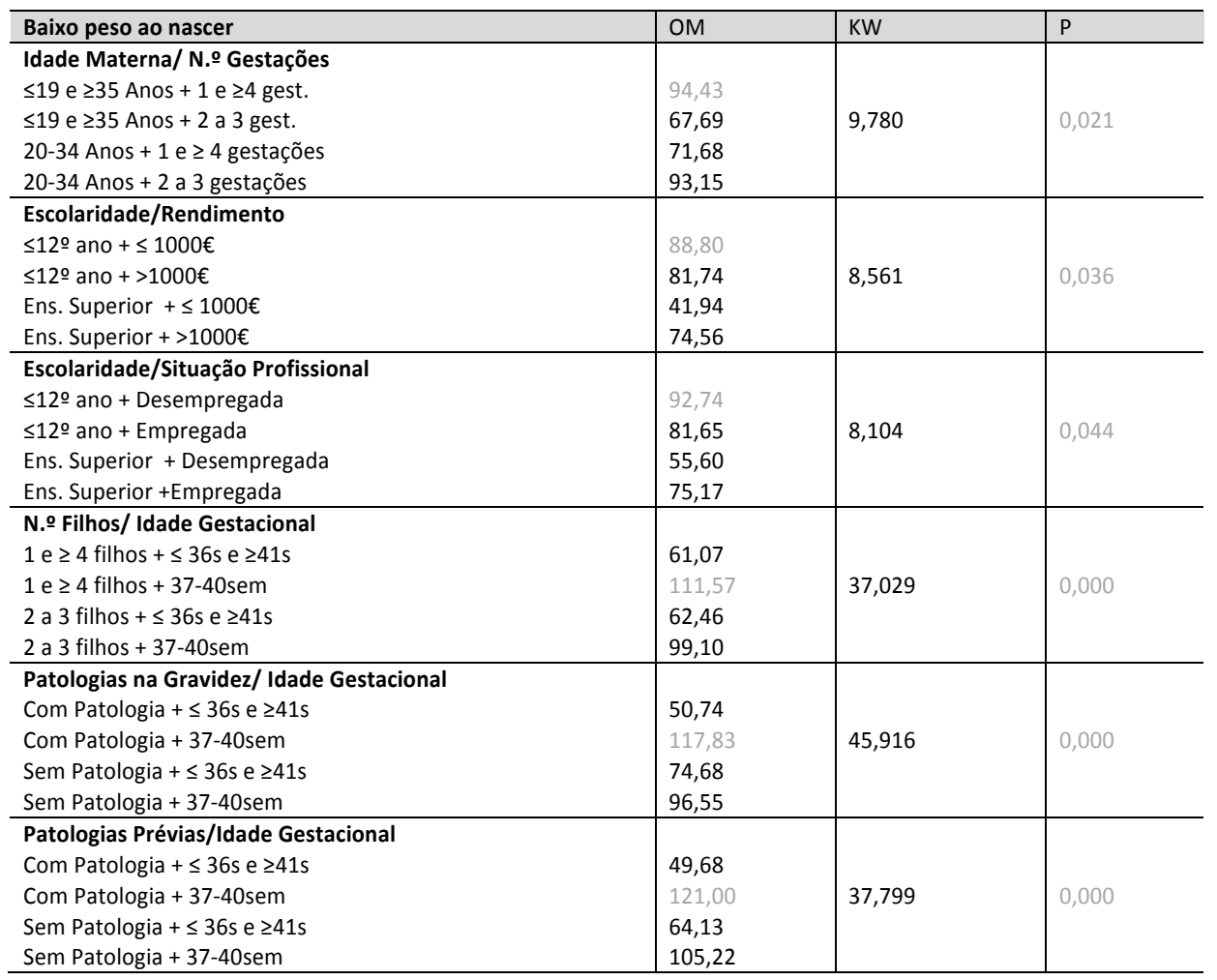

\section{DISCUSSÃO/CONCLUSÃO}

A definição da problemática do BPN, segundo Kramer(1987), é extremamente complexa e preenchida por múltiplos fatores causais ou não, tendo em conta que estes poderão variar de acor- 
do com a raça, realidade social, localização, meio ambiente, características genéticas, físicas, obstétricas e psicológicas da grávida.

Apurámos uma prevalência de BPN de 8,7\% correspondendo a 161 casos em 1846 puérperas inquiridas. Valores muito próximos ao nosso $(8,4 \%)$ também foram encontrados pelo INE(2012) no ano de 2011. De acordo com Thompson et al.(2005) a investigação da prevalência do BPN, tem que ter em conta o local onde é efetuado 0 estudo tal como as suas possíveis discrepâncias. A OMS revelou que entre 2000-2009 a taxa média europeia de BPN rondava os 7\%, sendo que Portugal estaria nos $8 \%$, contudo, esta taxa tem mostrado uma tendência crescente, de $7,2 \%$ em 2001, 8,2\% em 2009 e mais recentemente de 8,4\% em 2011. Segundo Machado (2011), tal facto poderá estar relacionado com a proporção de nascimentos em mães com idades superiores a 35 anos, ao uso de técnicas de fertilização e consequentemente também a um maior número de gestações múltiplas.

Relativamente ao BPN (8,7\%), esta investigação revelou que na nossa amostra (161), o maior número de casos apareceu no grupo etário entre os 20-34 anos (6\%). Os valores são muito próximos aos estimados pelo INE(2012) em 2011.

A incidência maior de aparecimento de BPN, calculada pela análise descritiva da variável, indica-nos que existe uma maior relação entre o BPN e as puérperas com idades $\leq 19$ anos, como de igual forma revelou Santos et al.(2009), contudo na análise inferencial não se comprovou associação, resultado este que se encontra em consonância com a revisão da literatura sobre o BPN efetuada por Prakeshkumar Shah(2008).

A baixa escolaridade é a condição com maior predominância na nossa amostra (66,9\%), e é também a esse nível que se associa uma maior incidência de BPN (10,425\%). A relação entre a escolaridade e o BPN surge, inicialmente, como estatisticamente significativa, e foi confirmada após ter sido testada (U Mann-Whitney) com uma significância marginal a 10\%. 0 mesmo resultado foi obtido por Carniel et al.(2008) que descreve que o nível de escolaridade influencia no interesse, e no deficit de conhecimentos relativos à gravidez.

Relativamente ao estado civil, a nossa investigação demonstrou que a maior proporção de casos de BPN surgiu associado às puérperas sem companheiro, apresentando um valor estatisticamente significativo nas mesmas. Alguns trabalhos justificam ainda que esta relação surge, porque muitas vezes a ausência de companheiro está relacionada à falta de adesão e à vigilância pré-natal (Coimbra et al., 2003). Após a aplicação de um Teste t de Student, não se confirmou a relação, tal como da mesma forma, foi concordante 0 estudo de Sullivan et al(2012), onde acrescenta que o estado civil é um fator muito fraco para o estabelecimento do BPN.

Atendendo à situação profissional, a nossa investigação vai de encontro à relação entre 0 BPN e o desemprego, surgindo com valores estatisticamente significativos na mesma, contudo não é confirmada na análise inferencial.

Normalmente 0 aparecimento de BPN surge como interligado ao baixo rendimento, uma vez que este poderá implicar deficits nutricionais, condições ambientais deficientes com provável aparecimento de doenças. Encontra-se ainda aliado a baixos níveis de instrução e profissões que podem conter risco apreciáveis para a saúde como ainda a analfabetismo e desemprego(Paula et al., 2010). 0 nosso estudo revelou que a proporção de aparecimento de BPN foi maior nas puérperas com baixos rendimentos mensais $(11,63 \%)$, apresentando diferença estatisticamente significativa nesse parâmetro. Testada a relação (t de Student), não se confirmou a influência desta variável no BPN, tal como 0 estudo efetuado por Vettore et al(2010).

Relativamente ao planeamento da gravidez, pressupõe-se que o não planeamento da mesma influencia no aparecimento do BPN, uma vez que poderá resultar numa vigilância tardia, adoção de comportamentos desajustados e ao não tratamento e estabilização de patologias prévias DGS (2006). 0 nosso estudo revelou que a proporção de BPN surgiu maioritariamente nas puérperas que 


\section{FATORES ASSOCIADOS AO BAIXO PESO AO NASCER}

não planearam a sua gravidez (10,09\%), mas sem demonstração de diferença estatisticamente significativa, como de igual forma aconteceu com Karaçam(2010). Após análise inferencial conseguiuse confirmar a influência entre as variáveis, com uma diferença estatística marginal a 10\%, o resultado foi concordante com Villalbí(2007).

Algumas patologias prévias e durante a gravidez poderão condicionar o ambiente intrauterino e afetar 0 crescimento fetal como também a duração da gestação(Ohlsson \& Shah, 2008). Relativamente ao nosso estudo a proporção de BPN foi maior nas puérperas que desenvolveram patologias $(13,49 \%)$ e também nas que já tinham história prévia de desenvolvimento de patologias $(11,54 \%)$. A análise inferencial revelou que, ambas patologias, prévias ou na gravidez, não interferiram no aparecimento de BPN tal como 0 estudo efetuado por Vettore et al.(2010).

Isto remete-nos a pensar que a assistência pré-natal efetuada a estas puérperas primou pela qualidade e eficácia no que concerne ao tratamento ou controle das suas patologias, ou que estas possam ter sido corrigidas atempadamente nas consultas de planeamento familiar.

A paridade, normalmente, surge fortemente associada à prematuridade nas primíparas e ao aparecimento de patologias durante a gravidez nas multíparas.

Na nossa investigação a análise das variáveis paridade, número de filhos e intervalo interparto não traduziu relevância significativa nas análises descritivas e inferenciais.

Segundo Machado et al(2011), em 2009 8,8\% dos nados vivos nasceram prematuramente. No estudo realizado, somente $8,5 \%$ das puérperas apresentaram recém-nascidos prematuros. Contudo é nessas puérperas que a proporção de BPN ultrapassa a estimada pelo autor do parágrafo anterior, sendo de $62,34 \%$. A análise estatística descritiva corrobora a interação da prematuridade com 0 BPN, como Carniel et al.(2008) revelou, contudo a inferencial já aponta, como sendo a que mais se relacionou, a idade gestacional pós-termo.

Contrariamente ao que nos diz a revisão bibliográfica, a gestação de termo, no nosso estudo surge como potenciadora do BPN quando em interação. A provável explicação para 0 aparecimento de gestações de termo como fator influenciador, pode ter relação com a adequada assistência pré-natal e controle de patologias ou condições que interfiram no aparecimento da prematuridade, mas não do BPN.

Segundo alguns autores a idade materna avançada e a multiparidade predispõem ao aumento de complicações tanto maternas como obstétricas e por consequência uma maior suscetibilidade ao BPN, nascimento pré-termo, RCIU, e intercorrências neonatais (Nogueira, 2010).

De acordo com Leal et al.(2006), a baixa escolaridade materna também se encontra associada à ocorrência de BPN. Para ele esta associação poderá estar relacionada com o elevado número de filhos que demonstram ter, sendo por vezes associado a um intervalo interparto inferior ao adequado, um baixo padrão socioeconómico ou ainda a situações de ausência de companheiro.

De acordo com Merck(2010), determinadas condições clínicas da mulher previamente à gravidez ou em gravidezes anteriores, poderão influenciar negativamente a gravidez atual. Segundo Silva(2009) o desenvolvimento de patologias durante a gravidez também pode incorrer num parto prematuro espontâneo, induzido ou cirúrgico, em função das complicações maternas ou fetais, influenciando desta forma, negativamente o peso ao nascer.

Concluindo a discussão, destaca referir que a etiologia multifactorial do BPN dificulta em muito a deteção dos fatores associados e por isso o estabelecimento de programas que possam resolver esta problemática.

Segundo Maia(2010), a realização de programas de assistência à saúde efetivos e de qualidade, podem contribuir para a redução do problema e das suas consequências. Contudo ações para a redução deste agravo extrapolam o setor de saúde, como constatado pela investigação realizada, e por isso exigem um esforço organizado de vários setores da sociedade. 
DESARROLLO PSICO-SOCIOEMOCIONAL DE LA EDAD:

PSICOLOGÍA POSITIVA Y BIENESTAR EN LAS PERSONAS MAYORES

\section{ACKNOWLEDGMENTS}

The Portuguese Foundation for Science and Technology (FCT) through the project PEstOE/CED/UI4016/2014, and the Centre for Studies in Education, Technologies and Health (CI\&DETS).

\section{REFERÊNCIAS BIBLIOGRÁFICAS}

Carniel, E. d. F., Zanolli, M. d. L., Antônio, M. Â. R. d. G. M., \& Morcillo, A. M. (2008). Determinantes do baixo peso ao nascer a partir das Declarações de Nascidos Vivos. Revista Brasileira de Epidemiologia, 11, 169-179.

Coimbra, L. C., Silva, A. A. M., Mochel, E. G., Alves, M. T. S. S. B., Ribeiro, V. S., Aragão, V. M. F., \& Bettiol, H. (2003). Fatores associados à inadequação do uso da assistência pré-natal. Revista de Saúde Pública, 37, 456-462.

DGS. (2006). Prestação de cuidados pré-concepcionais- Circular Normativa n.․․ 2. Ministério da Saúde Retrieved from http://srsdocs.com/parcerias/normas/circulares/dgs/2006/cuidados _pre_concepcionais.pdf.

Eisenhauer, E., Uddin, D., Albers, P., Paton, S., \& Stoughton, R. (2011). Establishment of a Low Birth Weight Registry and Initial Outcomes. Maternal \& Child Health Journal, 15(7), 921-930. doi: 10.1007/s10995-009-0540-8

INE. (2012). Nados-vivos (N.ํ) por Local de residência da mãe, Sexo, Local de parto da mãe, Assistência médica e Escalão de peso à nascença; Anual - INE, Nados-Vivos. from INE http://www.ine.pt/xportal/xmain?xpid=INE\&xpgid=ine_indicadores\&indOcorrCod=0005948\&co ntexto=bd\&selTab=tab2

Karaçam, Z., en, E., \& Amanak, K. (2010). Effects of unplanned pregnancy on neonatal health in Turkey: A case-control study. International Journal of Nursing Practice, 16(6), 555-563. doi: 10.1111/j.1440-172X.2010.01881.x

Kilsztajn, S., Rossbach, A., Carmo, M. S. N. d., \& Sugahara, G. T. L. (2003). Assistência pré-natal, baixo peso e prematuridade no Estado de São Paulo, 2000. Revista de Saúde Pública, 37, 303310. [citedIDate accessedI]. Retrieved rom` IWebsite Title website: IURLI doi:DOI

Kramer, M. S. (1987). Determinants of low birth weight: methodological assessment and metaanalysis. World Health Organization, 65( (5)), 663-737.

Lasker, J. N., Coyle, B., Li, K., \& Ortynsky, M. (2005). Assessment of risk factors for low birth weight deliveries. Health Care for Women International, 26(3), 262-280.

Leal, M. d. C., Gama, S. G. N. d., \& Cunha, C. B. d. (2006). Desigualdades sociodemográficas e suas conseqüências sobre o peso do recém-nascido. Revista de Saúde Pública, 40, 466-473.

Maia, R. d. R. P., \& Souza, J. M. P. d. (2010). Fatores associados ao baixo peso ao nascer em município do norte do Brasil. Revista brasileira de crescimento e desenvolvimento humano, 20, 735744.

Maria do Céu Machado, Maria Isabel Alves, \& Couceiro, M. L. (2011). Saúde Infantil e Juvenil em Portugal: indicadores do Plano Nacional de Saúde. Acta Pediátrica Portuguesa, 42(5), 195-204.

Merck. (2010). Manual Merck de Informação Médica: Saúde Para a Família. In 2 (Series Ed.) Broca (Ed.) (pp. 1944). Retrieved from http://www.manualmerck.net/

Nogueira, J. M. P. (2010). Factores associados ao Baixo Peso à Nascença. Universidade do Porto. Retrieved from http://repositorio-aberto.up.pt/bitstream/10216/54473/1/139111_ 1058TCD58.pdf

Ohlsson, A., \& Shah, P. (2008). Determinants and Prevention of Low Birth Weight: A Synopsis of the Evidence: The Institute of Health Economics.

Paula, C. G. D., Boccolini, C. S., Silva, A. A. M., Bacelo, A. C., Cardoso, F. T., \& Capelli, J. D. C. S. (2010). Baixo Peso ao Nascer: Fatores Socioeconômicos, Assistência Pré-Natal e Nutricional - 
Uma Revisão. Revista Augustus, 14(29), 54-65.

Santos, G. H. N. d., Martins, M. d. G., Sousa, M. d. S., \& Batalha, S. d. J. C. (2009). Impacto da idade materna sobre os resultados perinatais e via de parto. Revista Brasileira de Ginecologia e Obstetrícia, 31, 326-334.

Shah, P. S. (2010). Paternal factors and low birthweight, preterm, and small for gestational age births: a systematic review. Am J Obstet Gynecol, 202(2), 103-123.

Silva, E. S. N., \& Lahorgue, M. (2005). The influence of gestational age and birth weight in the clinical assesment of the muscle tone of healthy term and preterm newborns. Arquivos de NeuroPsiquiatria, 63, 956-962.

Silva, J. L. d. C. P. e., \& Surita, F. G. d. C. (2009). Idade materna: resultados perinatais e via de parto. Revista Brasileira de Ginecologia e Obstetrícia, 31, 321-325.

Sullivan, K., Raley, R. K., Hummer, R. A., \& Schiefelbein, E. (2012). The potential contribution of marital-cohabitation status to racial, ethnic, and nativity differentials in birth outcomes in Texas. Maternal And Child Health Journal, 16(4), 775-784.

Thompson, L. A., Goodman, D. C., Chiang-Hua, C., \& Stukel, T. A. (2005). Regional Variation in Rates of Low Birth Weight. [Article]. Pediatrics, 116(5), 1114-1121. doi: 10.1542/peds.20041627

Vettore, M. V., Gama, S. G. N. d., Lamarca, G. d. A., Schilithz, A. O. C., \& Leal, M. d. C. (2010). Housing conditions as a social determinant of low birthweight and preterm low birthweight. Revista de Saúde Pública, 44, 1021-1031.

Villalbí, J. R., Salvador, J., Cano-Serral, G., Rodríguez-Sanz, M. C., \& Borrell, C. (2007). Maternal smoking, social class and outcomes of pregnancy. Paediatric and perinatal Epidemiology, 21(5), 441-447.

WHO. (2011). World Health Statistics 2011. World Health Organization, 170. 